\title{
Restauração ecológica em áreas de mineração por cascalho no Sul do Brasil
}

A prática de restauração florestal vem se tornando cada vez mais necessária, visto que a perda da superfície florestal, ocasionada principalmente por atividades antrópicas, tem deixado a paisagem altamente fragmentada. Foi avaliada neste estudo a evolução do processo de restauração ecológica a partir de técnicas de nucleação em duas áreas com condições de solo distintas (com e sem deposição de resíduo de origem florestal) pós extração mineral de cascalho. A hipótese é que as técnicas de nucleação aplicadas são eficientes, bem como, o uso positivo do resíduo florestal, sendo uma metodologia promissora para a restauração de áreas mineradas por cascalho. Foram avaliadas três técnicas de nucleação (Galharia, Poleiro e Transposição do solo) e a regeneração natural em ambas as áreas, sendo que em uma delas ocorreu a deposição do resíduo de origem florestal antes da implantação das técnicas. Foi possível concluir que as áreas estão evoluindo e que a utilização do resíduo foi um método eficiente e atua de forma benéfica para a sucessão ecológica do ambiente. Porém, é importante destacar que a aplicação das técnicas de nucleação são as principais responsáveis pela constante evolução dessas áreas. Mesmo com resultados satisfatórios, vale ressaltar que é necessário a continuação do monitoramento nestas áreas.

\section{Ecological restoration in gravel mining areas in Southern Brazil}

\begin{abstract}
The practice of forest restoration has become increasingly necessary, since the loss of forest surface, caused mainly by human activities, has left the landscape highly fragmented. In this study, the evolution of the ecological restoration process using nucleation techniques in two areas with different soil conditions (with and without deposition of forest residues) was evaluated after mineral gravel extraction. The hypothesis is that the applied nucleation techniques are efficient, as well as, the positive use of forest waste, being a promising methodology for the restoration of areas mined by gravel. Three nucleation techniques (Gallaria, Perch and Soil Transposition) and natural regeneration in both areas were evaluated, in which one deposition of forest residues occurred before the implementation of the techniques. It was possible to conclude that the areas are evolving and that the use of the waste is an efficient method and acts in a beneficial way for the ecological succession of the environment. However, it is important to highlight that the application of nucleation techniques are mainly responsible for the constant evolution of these areas. Even with satisfactory results, it is noteworthy that continued monitoring in these areas is necessary.
\end{abstract}

Keywords: Gravel extraction deposit; Recovery of degraded areas; Natural regeneration; Forest waste; Nucleation techniques.

Topic: Ciências Florestais

Reviewed anonymously in the process of blind peer
Received: 03/07/2021

Approved: $27 / 07 / 2021$
Taynara Oliveira de Liz Buss (iD

Universidade do Estado de Santa Catarina, Brasil http://lattes.cnpq.br/8655311608969454 http://orcid.org/0000-0003-4958-429X

taynaradeliz@yahoo.com.br

\section{Maria Raquel Kanieski}

Universidade do Estado de Santa Catarina, Brasil http://lattes.cnpq.br/8826552075228927 http://orcid.org/0000-0003-1078-5641 raquel.kanieski@udesc.br

\section{Marcos Felipe Nicoletti (iD}

Universidade do Estado de Santa Catarina, Brasil http://lattes.cnpq.br/5635348559784763 http://orcid.org/0000-0003-4732-0119 marcos.nicoletti@udesc.br

\author{
Mariana de Moraes Goulart (iD) \\ Universidade do Estado de Santa Catarina, Brasil \\ http://lattes.cnpq.br/8946666429001234 \\ http://orcid.org/0000-0002-3666-2052 \\ marianamoraesgoulart@gmail.com \\ Daiany Augusta Paes Martins (id) \\ Universidade do Estado de Santa Catarina, Brasil \\ http://lattes.cnpq.br/2684777890431893 \\ http://orcid.org/0000-0001-5186-8107 \\ dapmflorestal@gmail.com

\section{Mireli Moura Pitz Floriani (iD} \\ Universidade do Estado de Santa Catarina, Brasil \\ http://lattes.cnpq.br/4094385791831130 \\ http://orcid.org/0000-0003-0067-1042 \\ mmpitz@klabin.com.br
}

Daniele Lourenço Souza (iD

Universidade do Estado de Santa Catarina, Brasi http://lattes.cnpq.br/8690319083710052 http://orcid.org/0000-0002-5870-2641 danielelourencosouza@gmail.com

\section{Juliane Dal Pizzol}

Universidade do Estado de Santa Catarina, Brasil http://lattes.cnpq.br/9356510516985849 http://orcid.org/0000-0001-9201-2831 judpizzol@gmail.com

\section{Charline Zangalli ic}

Universidade do Estado de Santa Catarina, Brasil http://lattes.cnpq.br/0983972588654675 http://orcid.org/0000-0002-7667-3389 charlineeng@gmail.com

\section{Referencing this:}

BUSS, T. O. L.; KANIESKI, M. R.; NICOLETTI, M. F.; GOULART, M. M.; MARTINS, D. A. P.; FLORIANI, M. M. P.; SOUZA, D. L.; PIZZOL, J. D.; ZANGALLI, C.. Restauração ecológica em áreas de mineração por cascalho no Sul do Brasil. Revista Ibero Americana de Ciências Ambientais, v.12, n.7, p.81-92, 2021. DOI: http://doi.org/10.6008/CBPC2179-6858.2021.007.0008 


\section{INTRODUÇÃO}

A crescente demanda por regularização ambiental da atividade mineradora tem feito com que estudos de restauração ecológica tragam resultados consistentes sobre métodos que auxiliam no aumento da diversidade e riqueza de espécies e que proporcionam maior cobertura do solo, com menor custo de implantação e manutenção. A restauração ecológica é a prática que desencadeia, facilita ou acelera a recuperação de ecossistemas alterados, permitindo adaptações às mudanças locais e globais, bem como a persistência e evolução de suas espécies componentes (GANN et al., 2019; SER, 2004). Sítios degradados pela ação da mineração apresentam baixa resiliência, pois o solo é totalmente retirado, tornando-se ausente de diásporos (sementes, caules, raízes ou tubérculos). Sendo assim, é importante que cada local perturbado tenha a chance e o tempo necessário para sua recuperação e que ações antrópicas auxiliem nesse processo (DUNAISKI et al., 2018).

A metodologia da nucleação na restauração ecológica tem por objetivo a formação de pequenos núcleos de diversidade, que representa a criação de uma nova condição na área degradada. Esta condição é resultado da combinação de diversos elementos, como por exemplo, sementes, solo, serapilheira, micro e macrobiota, que são retirados de remanescentes florestais próximos e incorporados nas áreas a serem restauradas (REIS et al., 2014). A aplicação conjunta de várias técnicas nucleadoras produzem uma diversidade de fluxos naturais na área degradada (ESPÍNDOLA et al., 2005), contribuindo para resgatar a funcionalidade dos sistemas naturais, aumentar a rugosidade do local e a conectividade da paisagem (REIS et al., 2014; CORBIN et al., 2012). Por normalmente apresentarem baixo custo de implantação, o emprego da nucleação é de grande valia para o processo de restauração.

As técnicas de nucleação têm sido recomendadas e aplicadas por cientistas, agências governamentais e empresas florestais do Brasil (TRENTIN et al., 2018; BECHARA et al., 2016; VOGEL et al., 2015; MARCUZZO et al., 2013). A avaliação e o monitoramento de áreas que sofreram mudanças estruturais em ambientes secundários é uma etapa fundamental e decisiva em projetos de restauração ecológica, pois indicam a eficácia das ações de restauração e, se necessário, é possível redefinir as metodologias utilizadas (BRANCALION et al., 2012).

Além disso, estudos com a utilização de resíduos florestais em áreas pós-mineração estão sendo realizados com o objetivo de auxiliar de forma positiva o avanço das técnicas nucleadoras, onde ocorre a reposição do substrato anteriormente eliminado buscando a revegetação da área para recuperação de seu equilíbrio ambiental. Porém, ainda existe uma enorme lacuna sobre a resposta do ambiente em relação ao resíduo. É de grande importância a verificação da aplicação destes resíduos, considerando a alta concentração de matéria orgânica, componente fundamental para o desenvolvimento de espécies arbóreas/arbustivas, e a possibilidade do uso alternativo deste resíduo para recuperação de áreas degradadas.

O objetivo deste estudo foi analisar a evolução do processo de restauração ecológica a partir de técnicas de nucleação em duas áreas com condições de solo distintas pós extração mineral de cascalho e 
verificar se a deposição de resíduo de origem florestal atua de forma positiva neste processo. Nossa hipótese é que as técnicas de nucleação aplicadas são eficientes, bem como, o uso positivo do resíduo florestal, sendo uma metodologia promissora para a restauração de áreas mineradas por cascalho.

\section{MATERIAIS E MÉTODOS}

\section{Caracterização geral da área de estudo}

Este estudo foi conduzido em duas áreas degradadas por atividades de mineração de cascalho, denominadas Westarp $\left(27^{\circ} 32^{\prime} 13^{\prime \prime}\right.$ S e $50^{\circ} 05^{\prime} 86^{\prime \prime}$ O) e Lauro $\left(27^{\circ} 13^{\prime} 36^{\prime \prime}\right.$ S e $50^{\circ} 07^{\prime} 08^{\prime \prime}$ O), localizadas no município de Otacílio Costa - SC, pertencentes a uma empresa do setor florestal.

As formações geológicas destas áreas remontam o Paleozóico com rochas sedimentares. O solo é classificado como Cambissolo Húmico Alumínico, considerado como solo pouco profundo ou raso, com cores de bruno escuro a bruno avermelhado, argiloso e com horizonte B incipiente (EMBRAPA, 2013), apresentam baixa fertilidade e alta pedregosidade.

O clima na região, conforme Alvares et al. (2013), é classificado como "Cfb", ou seja, temperado constantemente úmido, não apresentando estação seca definida com temperatura média anual em torno de 17,8 으. A precipitação anual é de $1.533 \mathrm{~mm}$ bem distribuídas ao longo do ano, com geadas frequentes (SCHICK et al., 2014; SILVA et al., 2012). A vegetação florestal pertence à Floresta Ombrófila Mista (IBGE, 2012), e na adjacência há plantio de espécies exóticas dos gêneros Pinus e Eucalyptus.

A área experimental Westarp possui $2.500 \mathrm{~m}^{2}$, já a Lauro possui $1.500 \mathrm{~m}^{2}$. Ambas foram degradadas em função da abertura de estradas para acessar as cascalheiras e eram utilizadas para manobrar maquinários em virtude da mineração de cascalho para a pavimentação de estradas florestais. O solo na Westarp é argiloso com muitos fragmentos de rocha e compactação excessiva. Em contrapartida, na Lauro no ano de 2015, houve a deposição de resíduo florestal casca de Pinus spp. misturados com resíduo mineral de cascalho, no qual foram depositados em leiras, espalhados e compactados com trator de esteira.

\section{Técnicas de nucleação}

Foram instaladas 32 unidades amostrais na Westarp e 19 na Lauro, no ano de 2015, dispostas de forma aleatória, compostas pelas técnicas nucleadoras poleiro artificial, transposição de serapilheira e do banco de sementes do solo e enleiramento de galharia. Parcelas de condução da regeneração natural também foram implantadas nas entrelinhas das técnicas, totalizando sete unidades amostrais em cada área.

Tendo por finalidade atrair a avifauna com intuito de auxiliar no processo de recolonização da área, foram instalados poleiros artificiais os quais foram inseridos em parcelas com dimensões de $1 \mathrm{~m} \times 1 \mathrm{~m}$, com altura aproximada de $2,5 \mathrm{~m}$. Na Westarp havia sete unidades amostrais e na Lauro seis. Para a transposição de serapilheira e do banco de sementes do solo foram alocadas 15 unidades amostrais na Westarp e sete na Lauro, com dimensões de $1 \mathrm{~m}$ x $1 \mathrm{~m}$. Na Westarp os núcleos com enleiramento de galharia incluíram dez pilhas e na Lauro seis, com área média de $2,5 \mathrm{~m}^{2}(1,5 \mathrm{~m} \times 1,5 \mathrm{~m})$ e aproximadamente $0,50 \mathrm{~m}$ de altura. Foram 
distribuídas aleatoriamente entre as técnicas de nucleação as parcelas de condução da regeneração natural de $1 \mathrm{~m}$ x $1 \mathrm{mErro!} \mathrm{Fonte} \mathrm{de} \mathrm{referência} \mathrm{não} \mathrm{encontrada..}$

As avaliações foram realizadas em duas etapas, a primeira em 2016, um ano após a implantação do experimento, e a segunda em 2018, três anos após a implantação. Estas avaliações tanto para as técnicas de nucleação, como para parcelas de regeneração natural consistiu na identificação e quantificação dos indivíduos regenerantes recrutados dentro das parcelas, sem critério de inclusão, sendo eles demarcados com cordão colorido.

\section{Análise de dados}

Todos os indivíduos identificados foram classificados de acordo com a forma de vida, grupo ecológico e síndrome de dispersão. A riqueza entre as áreas de estudo foi comparada por meio do método de rarefação. A diversidade da composição florística foi realizada para as áreas Lauro e Westarp, nos anos de 2016 e 2018, a partir do índice de Shannon-Wiener $\left(H^{\prime}\right)$ e da equabilidade de Pielou $(J)$ que representa a uniformidade da distribuição dos indivíduos entre as espécies existentes (BROWER et al., 1984). As análises dos dados foram realizadas no programa estatístico R (R CORE TEAM, 2019) utilizando-se do pacote Vegan (OKSANEN et al., 2019).

\section{RESULTADOS}

Na primeira avaliação (2016) foi obtido um total de 3.642 indivíduos regenerantes, incluindo as unidades amostrais de regeneração natural, de 19 famílias, 85 espécies e 53 gêneros. Desses, 12 foram identificados somente ao nível de gênero e 13 espécies não foram identificadas (Tabela 1). Dentro das espécies não identificadas foram contabilizados 21 indivíduos na área Westarp e 12 na área Lauro. Na área Westarp o número de indivíduos totalizou 2.489, distribuídos em 53 espécies. Já na área Lauro foram quantificados 1.153 indivíduos dentro de 63 espécies.

Tabela 1: Relação de famílias, espécies e número de indivíduos regenerantes nas técnicas nucleadoras e da regeneração natural nas áreas Lauro (L) e Westarp (W), nas avaliações de 2016 e 2018.

\begin{tabular}{|c|c|c|c|c|c|c|c|}
\hline Família/ Espécie & $\mathrm{FV}$ & SD & $\mathrm{GR}$ & L2016 & L2018 & W2016 & W2018 \\
\hline \multicolumn{8}{|l|}{ APIACEAE } \\
\hline Apiaceae 1 & $\mathrm{Nc}$ & Nc & Nc & 0 & 0 & 0 & 11 \\
\hline Centella asiatica (L.) Urb. & $\mathrm{Hb}$ & Aut & Si & 25 & 285 & 7 & 57 \\
\hline Cyclospermum leptophyllum (Pers.) Sprague ex Britton \& P.Wilson & $\mathrm{Hb}$ & Ane & $P$ & 41 & 75 & 0 & 0 \\
\hline \multicolumn{8}{|l|}{ ASTERACEAE } \\
\hline Achyrocline satureioides (Lam.) DC. & $\mathrm{Hb}$ & Ane & $P$ & 0 & 1 & 181 & 407 \\
\hline Asteraceae 1 & $\mathrm{Nc}$ & $\mathrm{Nc}$ & $\mathrm{Nc}$ & 0 & 4 & 0 & 1 \\
\hline Asteraceae 2 & $\mathrm{Nc}$ & $\mathrm{Nc}$ & $\mathrm{Nc}$ & 0 & 2 & 0 & 0 \\
\hline Baccharis sp. 1 & Arb & Ane & $\mathrm{Nc}$ & 0 & 1 & 0 & 0 \\
\hline Baccharis sp. 2 & Arb & Ane & $\mathrm{Nc}$ & 0 & 17 & 0 & 10 \\
\hline Baccharis dracunculifolia DC. & Arb & Ane & $\mathrm{P}$ & 19 & 28 & 86 & 572 \\
\hline Baccharis semiserrata DC. & Arb & Ane & $\mathrm{P}$ & 0 & 5 & 0 & 35 \\
\hline Baccharis trimera (Less.) DC. & Arb & Ane & Si & 0 & 1 & 18 & 56 \\
\hline Baccharis uncinella DC. & Arb & Ane & $\mathrm{P}$ & 20 & 11 & 111 & 116 \\
\hline Baccharis vulneraria Baker. & Arb & Ane & $P$ & 0 & 0 & 25 & 0 \\
\hline Chaptalia nutans (L.) Pol. & $\mathrm{Hb}$ & Ane & St & 3 & 68 & 0 & 0 \\
\hline Chevreulia sarmentosa (Pers.) Blake & $\mathrm{Hb}$ & Ane & $\mathrm{Si}$ & 32 & 89 & 14 & 101 \\
\hline Conyza bonariensis (L.) Cronquist & $\mathrm{Hb}$ & Ane & $\mathrm{P}$ & 167 & 213 & 0 & 297 \\
\hline
\end{tabular}


Eclipta prostrata (L.) L.

Erechtites valerianifolius (Link ex Spreng.) DC.

Gamochaeta pensylvanica (Willd.) Cabrera

Gamochaeta stachydifolia (Lam.) Cabrera

Hypochaeris radicata L.

Senecio brasiliensis (Spreng.) Less.

Sonchus oleraceus L.

Vernonanthura sp.1

Vernonanthura tweediana (Baker) H. Rob.

Vernonia sp. 1

BERBERIDACEAE

Berberis laurina Billb.

CARYOPHYLLACEAE

Spergula sp. 1

COMMELINACEAE

Commelina sp.

CONVOLVULACEAE

Convolvulaceae 1

CYPERACEAE

Bulbostylis capillaris (L.) C.B.Clarke

Bulbostylissp. 1

Carex brasiliensis A.St.-Hil.

Cyperus aggregatus (Willd.) Endl.

Cyperus agrestis Willd. ex Spreng. \& Link

Cyperus odoratus L.

Cyperus rigens C.Presl

Fimbristylis dichotoma (L.) Vahl

Kyllinga brevifolia Rottb.

Rhynchospora barrosiana Guagl.

EUPHORBIACEAE

Croton triqueter Lam.

Sapium glandulosum (L.) Morong

FABACEAE

Desmodium adscendens (Sw.) DC.

Fabaceae 1

Medicago lupulina $\mathrm{L}$.

Mimosa scabrella Benth.

Senna macranthera (DC. ex Collad.) H.S.Irwin \& Barneby

Senna neglecta (Vogel) H.S.Irwin \& Barneby

Trifolium polymorphum Poir.

Trifolium repens $\mathrm{L}$.

HYMENOPHYLLACEAE

Trichomanes sp. 1

IRIDACEAE

Sisyrinchium sp. 1

Sisyrinchium micranthum Cav.

Sisyrinchium cf. vaginatum

JUNCACEAE

Juncus microcephalus Kunth

LAURACEAE

Lauraceae 1

LYTHRACEAE

Cuphea carthagenensis (Jacq.) J.F.Macbr.

MALVACEAE

Sida rhombifolia L.

Sida sp. 1

MELASTOMATACEAE

Leandra australis (Cham.) Cogn.

Leandra sp. 1

INDETERMINADA

Indeterminadas*

ONAGRACEAE

Ludwigia sp.1

PINACEAE

Pinus sp.

PLANTAGINACEAE

$\begin{array}{llll}\mathrm{Hb} & \text { Ane } & \mathrm{St} & 3 \\ \mathrm{Hb} & \text { Ane } & \mathrm{P} & 53 \\ \mathrm{Hb} & \text { Ane } & \mathrm{P} & 24 \\ \mathrm{Hb} & \text { Ane } & \mathrm{P} & 9 \\ \mathrm{Hb} & \text { Ane } & \mathrm{Si} & 36 \\ \mathrm{Arb} & \text { Ane } & \mathrm{P} & 79 \\ \mathrm{Hb} & \text { Ane } & \mathrm{P} & 28 \\ \mathrm{Nc} & \text { Ane } & \mathrm{Nc} & 0 \\ \mathrm{Arb} & \text { Ane } & \mathrm{P} & 0 \\ \mathrm{Nc} & \mathrm{Nc} & \mathrm{Nc} & 0\end{array}$

$0 \quad 0 \quad 0$

$\begin{array}{lll}152 & 22 & 96\end{array}$

$32 \quad 103 \quad 14$

23
$50-13$

$13 \quad 108$

$\begin{array}{lll}60 & 0 & 48\end{array}$

$\begin{array}{lll}0 & 0\end{array}$

$\begin{array}{lll}3 & 9 & 0\end{array}$

$\begin{array}{lll}0 & 28 & 0\end{array}$

$\begin{array}{lllllll}\text { Arb Zoo } & \mathrm{P} & 0 & 0 & 0 & 4\end{array}$

$\begin{array}{lllllll}\text { Nc } & \text { Nc } & \text { Nc } & 0 & 6 & 0 & 52\end{array}$

$\begin{array}{lllllll}\mathrm{Nc} & \mathrm{Nc} & \mathrm{Nc} & 6 & 9 & 0 & 22\end{array}$

$\begin{array}{lllllll}\text { Nc } & \text { Nc } & \text { Nc } & 0 & 63 & 0 & 0\end{array}$

$\begin{array}{lllllll}\mathrm{Hb} & \text { Ane } & \mathrm{P} & 4 & 17 & 54 & 28\end{array}$

$\mathrm{Nc} \quad \mathrm{Nc} \quad \mathrm{Nc} \quad 0 \quad 0001$

$\mathrm{Hb}$ Ane $\mathrm{P} \quad 0 \quad 13 \quad 0 \quad 0$

$\mathrm{Hb}$ Ane $\mathrm{P} \quad 0 \quad 30 \quad 003$

$\mathrm{Hb}$ Ane $\mathrm{P} \quad 0 \quad 15 \quad 000$

$\begin{array}{lllllll}\mathrm{Hb} & \text { Ane } & \mathrm{P} & 38 & 7 & 48 & 1\end{array}$

$\mathrm{Hb}$ Ane $\mathrm{P} \quad 13 \quad 2 \quad 000$

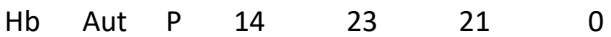

$\mathrm{Hb}$ Aut $\mathrm{P} \quad 0 \quad 0000$

$\begin{array}{lllllll}\mathrm{Hb} & \text { Aut } & \mathrm{P} & 8 & 2 & 7 & 0\end{array}$

$\begin{array}{lllllll}\text { Arb } & \text { Aut } & \mathrm{P} & 8 & 17 & 0 & 0\end{array}$

Arv Aut $\begin{array}{lllll}\mathrm{Si} & 3 & 4 & 0 & 0\end{array}$

$\begin{array}{lllllll}\mathrm{Hb} & \text { Zoo } & \mathrm{Si} & 0 & 29 & 8 & 17\end{array}$

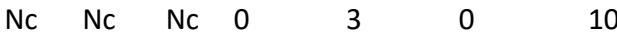

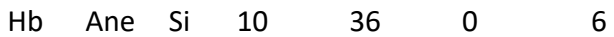

Arv Aut $\mathrm{P} \quad 0 \quad 001$

Arv Aut $\mathrm{P} \quad 0 \quad 018 \quad 0 \quad 0$

Arb Aut $\mathrm{P} \quad 0 \quad 32 \quad 0 \quad 0$

$\begin{array}{lllllll}\mathrm{Hb} & \text { Aut } & \mathrm{P} & 19 & 292 & 17 & 16\end{array}$

$\mathrm{Hb}$ Aut $\mathrm{P} \quad 0 \quad 114 \quad 0 \quad 5$

Nc Nc Nc $2020 \quad 0 \quad 0$

$\begin{array}{lllllll}\text { Nc } & \text { Nc } & \text { Nc } & 8 & 1 & 24 & 10\end{array}$

$\mathrm{Hb}$ Aut $\mathrm{P} \quad 8 \quad 0000$

$\mathrm{Hb}$ Ane $\mathrm{P} \quad 12 \quad 0 \quad 000$

$\begin{array}{lllllll}\mathrm{Hb} & \text { Aut } & \mathrm{P} & 0 & 0 & 49 & 0\end{array}$

Nc Nc Nc $\begin{array}{lllll} & 0 & 0 & 0 & 2\end{array}$

$\begin{array}{lllllll}\mathrm{Hb} & \text { Zoo } & \mathrm{Si} & 5 & 10 & 13 & 0\end{array}$

$\begin{array}{lllllll}\mathrm{Hb} & \text { Aut } & \mathrm{Si} & 2 & 42 & 35 & 49\end{array}$

$\mathrm{Hb}$ Aut $\mathrm{Nc} 3 \quad 0000$

$\begin{array}{lllllll}\text { Arb Ane } & \text { Si } & 0 & 6 & 0 & 0\end{array}$

$\begin{array}{llllll}\text { Arb Ane Nc } & 0 & 19 & 30 & 12\end{array}$

$\begin{array}{lllllll}\text { Nc } & \text { Nc } & \text { Nc } & 21 & 19 & 12 & 8\end{array}$

$\begin{array}{llllll}\text { Arb Ane Nc } & 0 & 5 & 0 & 43\end{array}$

Nc Ane Nc $0 \quad 0 \quad 0 \quad 2$ 
Plantago australis Lam.

Plantago sp. 1

Plantago tomentosa Lam.

POACEAE

Andropogon sp.

Andropogon bicornis L.

Andropogon leucostachyus Kunth

Anthoxanthum odoratum L.

Briza minor L.

Cortaderia selloana (Schult. \& Schult.f.) Asch. \& Graebn.

Cynodon dactylon (L.) Pers.

Dichanthelium sabulorum (Lam.) Gould \& C.A. Clark

Digitaria sp. 1

Digitaria sp. 2

Digitaria sp. 3

Eleusine indica (L.) Gaertn.

Eragrostis plana Nees

Eragrostis polytricha Nees

Panicum sp.1

Panicum sp.2

Panicum sp. 3

Paspalum dilatatum Poir.

Paspalum mandiocanum Trin.

Paspalum sp. 1

Paspalum urvillei Steud.

Poa annua $\mathrm{L}$.

Setaria parviflora (Poir.) Kerguélen

RUBIACEAE

Borreria latifolia (Aubl.) K.Schum.

Mitracarpus hirtus (L.) DC.

Richardia brasiliensis Gomes

RUTACEAE

Zanthoxylum rhoifolium Lam.

SOLANACEAE

Solanum americanum Mill.

Solanum mauritianum Scop.

Solanum pseudoquina A.St.-Hil.

Solanum sp. 1

Solanum viarum Dunal

VERBENACEAE

Verbena litoralis Kunth

Verbena sp. 1

VIOLACEAE

Hybanthus communis (A.St.-Hil.) Taub.

*Na avaliação de 2016, 13 espécies não foram determinadas e na avaliação de 2018, nove espécies. FV: forma de vida (Arb: arbustivo; Arv: arbóreo; Hb: herbáceo); SD: síndrome de dispersão (Ane: anemocoria; Aut: autocoria; Zoo: zoocoria); GE: Grupo ecológico (P: pioneira; Si: secundária inicial; St: secundária tardia). Nc: Não classificada.

As famílias mais representativas quanto à riqueza, em 2016, foram Poaceae (20 espécies), Asteraceae (18), Cyperaceae (seis) e Fabaceae (quatro). Os gêneros mais representativos foram Baccharis com quatro espécies, além de Digitaria, Eragrostis, Panicum, Paspalum, Sisyrinchium e Solanum, com três espécies cada. As famílias com maior destaque na Westarp são Poaceae (14 espécies), Asteraceae (13) e Cyperaceae (quatro) e, na Lauro Poaceae (17 espécies), Asteraceae (12) e Cyperaceae (cinco).

Na segunda avaliação das técnicas nucleadoras, em 2018, também incluindo as unidades amostrais de regeneração natural foram recrutados 10.696 indivíduos de 22 famílias botânicas, incluindo 97 espécies e 54 gêneros (Tabela 1). Além dessas, foram computadas nove espécies como indeterminadas, sendo oito indivíduos na área Westarp e 19 na Lauro, e cinco identificadas apenas em nível de família (Apiaceae, Asteraceae, Convolvulaceae, Fabaceae, Lauraceae). Nas áreas Westarp e Lauro o número de indivíduos 
recrutados foi de 7.130 e 3.566, respectivamente. A Lauro apresentou riqueza superior, com 84 espécies, enquanto a Westarp registrou 56 espécies.

Três famílias botânicas (Juncaceae, Rutaceae e Violaceae) e 13 espécies identificadas em 2016 não foram registradas em 2018. Em contrapartida, 29 novas espécies e 7.054 indivíduos ingressaram na comunidade. Apenas uma espécie que ingressou é exótica, Pinus sp. 1. Andropogon bicornis L. foi destaque do estudo com 3.766 indivíduos ingressados na área Westarp e 578 na Lauro, totalizando 4.344 indivíduos amostrados.

As famílias com maior riqueza, no ano de 2018, considerando as duas áreas são Asteraceae (20), Poaceae (19 espécies), Cyperaceae (10) e Fabaceae (oito). Os gêneros mais representativos foram Baccharis (seis espécies), Solanum (cinco), Cyperus (quatro) e Paspalum (quatro). As famílias com maior destaque na Westarp são Asteraceae (15 espécies), Poaceae (12) e Fabaceae (seis) e, na Lauro Asteraceae (20 espécies), Poaceae (18) e Cyperaceae (nove). Em relação à forma de vida, grupo ecológico e síndrome de dispersão os resultados encontrados foram semelhantes em ambas as avaliações (Tabela 2).

Tabela 2: Percentual de espécies em relação à forma de vida, grupo ecológico e síndrome de dispersão de cada área em seus respectivos anos de avaliação.

\begin{tabular}{|c|c|c|c|c|c|c|c|c|c|}
\hline \multirow{3}{*}{ Área/Ano } & \multicolumn{9}{|c|}{ Porcentagem de Espécies (\%) } \\
\hline & \multicolumn{3}{|l|}{ FV } & \multicolumn{3}{|l|}{$\mathrm{GE}$} & \multicolumn{3}{|l|}{ SD } \\
\hline & $\mathrm{Hb}$ & Arb & Arv & $\mathrm{P}$ & $\mathrm{Si}$ & St & Ane & Aut & Zoo \\
\hline Lauro 2016 & 82 & 14 & 4 & 80 & 16 & 4 & 56 & 30 & 14 \\
\hline Lauro 2018 & 73 & 24 & 3 & 79 & 19 & 2 & 59 & 27 & 14 \\
\hline Westarp 2016 & 77 & 20 & 3 & 76 & 24 & 0 & 50 & 38 & 12 \\
\hline Westarp 2018 & 72 & 23 & 5 & 80 & 20 & 0 & 62 & 28 & 10 \\
\hline
\end{tabular}

FV: forma de vida (Arb: arbustivo; Arv: arbóreo; Hb: herbáceo); GE: Grupo ecológico (P: pioneira; Si: secundária inicial; St: secundária tardia); SD: síndrome de dispersão (Ane: anemocoria; Aut: autocoria; Zoo: zoocoria).

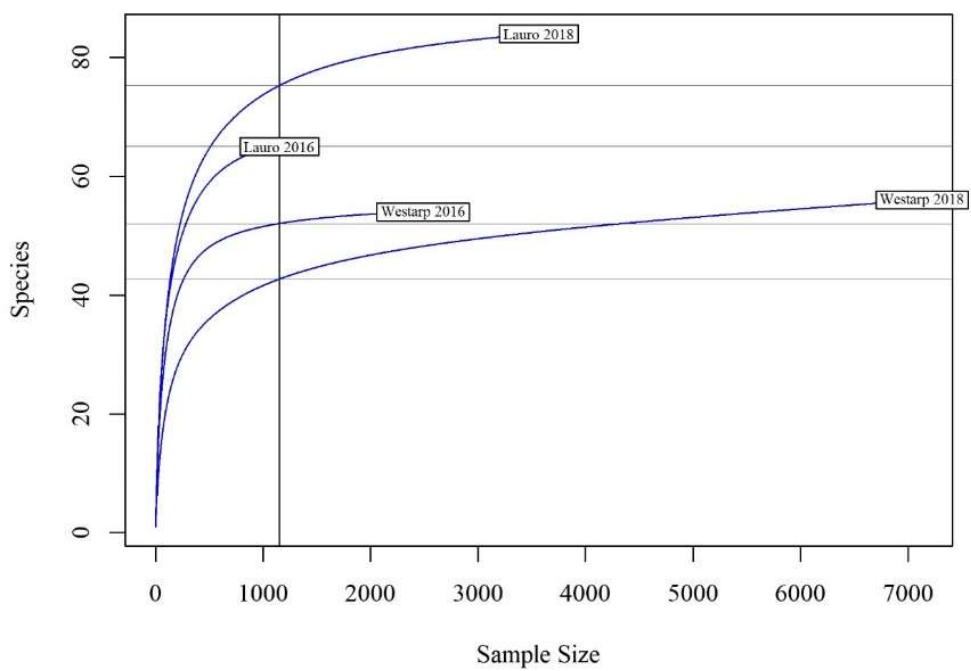

Figura 1: Curvas de acumulação de espécies, usando método de rarefação, para as áreas Lauro e Westarp, nos anos de 2016 e 2018, que estão em processo de restauração ecológica.

A comparação da riqueza de espécies resultantes da curva de rarefação por indivíduos (Figura 1) indicou diferença entre as duas áreas para o presente estudo. Com base na padronização da menor abundância de indivíduos levantada é possível verificar novamente que a área Lauro apresentou riqueza superior. Em comparação com os resultados de 2016, a área Lauro obteve um acréscimo de 21 espécies e a 
Westarp de três espécies em 2018.

Analisando o índice de diversidade de Shannon e a equabilidade de Pielou (Tabela 3), observa-se uma diminuição na área Westarp nos valores encontrados no ano de 2018 em relação ao ano de 2016. Em contrapartida, na Lauro ocorreu um acréscimo nos valores destes índices.

Tabela 3: Diversidade de Shannon ( $\left.\mathrm{H}^{\prime}\right)$, Equabilidade de Pielou (J), para as áreas Lauro e Westarp no ano de 2016 e 2018.

\begin{tabular}{lll}
\hline Área/Ano & $\mathrm{H}^{\prime}$ & $\mathrm{J}$ \\
\hline Lauro 2016 & 3,48 & 0,86 \\
Lauro 2018 & 3,54 & 0,80 \\
Westarp 2016 & 3,25 & 0,84 \\
Westarp 2018 & 2,16 & 0,54 \\
\hline
\end{tabular}

\section{DISCUSSÃO}

O ingresso de novas espécies demonstra que as técnicas implantadas durante o processo de restauração estão permitindo a superação dos filtros ecológicos. Estes filtros compreendem a uma série de razões pelas quais as espécies podem ou não ingressar e sobreviver num local (HOBBS et al., 2004) e eles podem atuar como barreiras, impedindo a continuidade da sucessão (ENGEL et al., 2003). Os filtros ecológicos são divididos em filtros abióticos (clima, substrato e estrutura da paisagem) e filtros bióticos (competição, predação, dispersão, mutualismo, distúrbios, ordem de chegada das espécies e remoção de espécies indesejáveis) (HOBB et al., 2004). Assim, é possível observar que a presença de espécie exótica, Pinus sp., na área é um filtro ecológico biótico que futuramente pode dificultar o desenvolvimento das espécies nativas e o sucesso do processo de restauração, uma vez que, esta espécie apresenta elevado potencial de invasão biológica principalmente em áreas abertas, antropizadas ou áreas de florestas em estágio inicial de regeneração (BECHARA et al., 2014). A junção destes fatores e a presença de plantios comerciais no entorno das áreas demonstram a necessidade do manejo de espécies exóticas invasoras nas áreas em estudo.

A maior riqueza das famílias Asteraceae e Poaceae nas áreas já era esperado, visto que nessas famílias estão incluídas espécies que comumente ocupam áreas abertas em processos iniciais de sucessão. Dunaiski Junior (2015), ao avaliar comunidades herbáceas e arbustivas na Floresta Ombrófila Mista em áreas degradadas pela mineração de calcário encontrou 97 espécies no sítio que estava há cinco anos em processo de regeneração e as famílias com maior riqueza também foram Asteraceae, Poaceae e Fabaceae. Ainda conforme o mesmo autor, a grande maioria das espécies encontradas por ele fazem parte da família Asteraceae, a qual juntamente com a família Poaceae, foram as famílias mais representativas no presente estudo.

Espécies dos gêneros Baccharis e Solanum são normalmente encontradas em maiores quantidades em áreas de estágio inicial de sucessão, pois são pioneiras, de rápido crescimento e se adaptam às condições do ambiente em que são submetidas. De acordo com Bechara et al. (2007) áreas com regeneração herbáceoarbustiva bem desenvolvida, proliferam-se por todo o ambiente, preparando o mesmo para os indivíduos subsequentes e atraindo fauna polinizadora e dispersora. Essas proliferações muitas vezes são compostas 
por aglomerados pioneiros de asteráceas, tal como Baccharis dracunculifolia. Fato que foi evidentemente observado no atual trabalho, em que se registrou número significativo de indivíduos (705) dessa espécie.

Ao trabalhar com restauração florestal em uma área de preservação permanente degradada em Santa Catarina, Rech et al. (2015) inferiu que as espécies do gênero Baccharis preferem áreas abertas ou alteradas, com alta incidência de luz, sugerindo assim o avanço da sucessão em áreas em estágio inicial de sucessão. Essa característica é essencial para a recuperação de áreas mineradas por cascalho, pois conforme Dunaiski Junior (2015), o repovoamento é desafiador até mesmo para as herbáceas pioneiras, devido à falta de matéria orgânica no solo e a aridez da superfície. Pereira et al. (2015) acrescenta que locais que sofreram exploração de cascalho apresentam condições adversas ao estabelecimento de espécies vegetais, devido principalmente a baixa disponibilidade hídrica e elevada compactação do solo.

A espécie com maior destaque, $A$. bicornis, é uma espécie nativa pertencente à família Poaceae. Segundo Neri et al. (2011) ela representa uma das herbáceas de maior importância no início do processo de sucessão secundária. Suas características de grande produção e longevidade de sementes, dispersão anemocórica, tolerância a locais antropizados, justificam sua alta presença nas áreas desta pesquisa. Além disso, esta espécie tem a particularidade de formar uma cobertura contínua no solo, sendo dominantes em áreas úmidas ou próximas a cursos d'água, em locais alterados e de cultivo abandonados e em afloramentos rochosos de decomposição mais avançada (ZANIN et al., 2011).

A forma de vida mais representativa das espécies foi a herbácea, o grupo ecológico mais expressivo foi o grupo das pioneiras e a síndrome de dispersão mais relevante foi a anemocórica. Trabalhos como o de Dunaiski Junior (2015), também registrou maior porcentagem de espécies herbáceas (78\%), seguido de espécies arbustivas (20\%) e arbóreas (2\%). Oliveira et al. (2018) dizem que é comum encontrar maior número de espécies herbáceas no início do processo de sucessão de áreas degradadas, mesmo quando em formações florestais.

Dunaiski Junior (2015) registrou na fase de cinco anos de regeneração de área minerada por calcário, 75\% de pioneiras e Rech et al. (2015) obtiveram para o grupo de indivíduos jovens o percentual de $23,9 \%$ de pioneiras ao avaliarem áreas de preservação permanente após seis anos de implantação da proteção da área, degradada pela presença de gado. O percentual de pioneiras do atual projeto é superior aos autores citados, salientando que se passaram apenas três anos da implantação da restauração ecológica, o que de fato confirma o estágio inicial de sucessão das áreas. Neste cenário a espécies anemocóricas foram as mais representativas e a implicação deste resultado deve-se ao fato das asteráceas estarem dominando o ambiente, as quais são espécies predominantemente dispersas pelo vento.

O destaque da Lauro perante Westarp, quanto riqueza de espécies, pode ser explicada pelo de o substrato formado por resíduo florestal casca de Pinus spp. misturados com resíduo mineral de cascalho proporcionar melhores condições, facilitando que espécimes mais exigentes e de estágios mais avançados de sucessão consigam ingressar na comunidade.

A diversidade florística, determinada pelo índice de diversidade de Shannon $\left(\mathrm{H}^{\prime}\right)$, indicou que a área 
Lauro $\left(H^{\prime}: 3,54\right)$ possui maior diversidade que a área Westarp $\left(H^{\prime}: 2,16\right)$. Além disso, em 2016 a Westarp apresentou maior diversidade que no ano de 2018, essa diminuição é justificada, principalmente, pela presença abundante de indivíduos da A. bicornis.

Em estudos como o de Dunaiski Junior (2015), foi encontrado um valor de Shannon de $H^{\prime}$ : 3,30, em uma área em processo de regeneração há cinco anos pós mineração de calcário, sendo muito próximo ao valor obtido na Lauro. Infere-se nestes casos que em três anos esta área está evoluindo no processo de restauração de forma satisfatória. Starr et al. (2012) ao avaliar o efeito de dois tipos de manejo em área minerada por cascalho no Distrito Federal registrou na área revegetada com Stylosanthes spp. $H^{\prime}=2,20$, e na área em que não houve tratamento do substrato $H^{\prime}=1,50$. Esse último resultado pode ser equiparado à Westarp registrando ainda maior diversidade mesmo apresentando-se com solo descoberto, confirmando a importância de se obter como primeira medida da recuperação a cobertura do solo em áreas degradadas pela mineração de cascalho.

Perante a equabilidade de Pielou, foi possível observar que a área Lauro relatou a maior equalibidade em ambos os anos de avaliação, indicando que a área é composta por espécies semelhantemente abundantes, não ocorrendo uma única ou poucas espécies que dominam o local. Porém, a menor equabilidade na área Westarp, em 2018, indica que existem menos espécies e que algumas delas concentram a maior quantidade de regenerantes. Este índice ratifica que a Lauro se apresenta com boa cobertura de solo, alta diversidade e abundância de espécies, confirmando que a alternativa de inserir resíduo florestal sobre áreas degradadas pela mineração de cascalho é benéfica para a sucessão ecológica do ambiente (Figura 2).

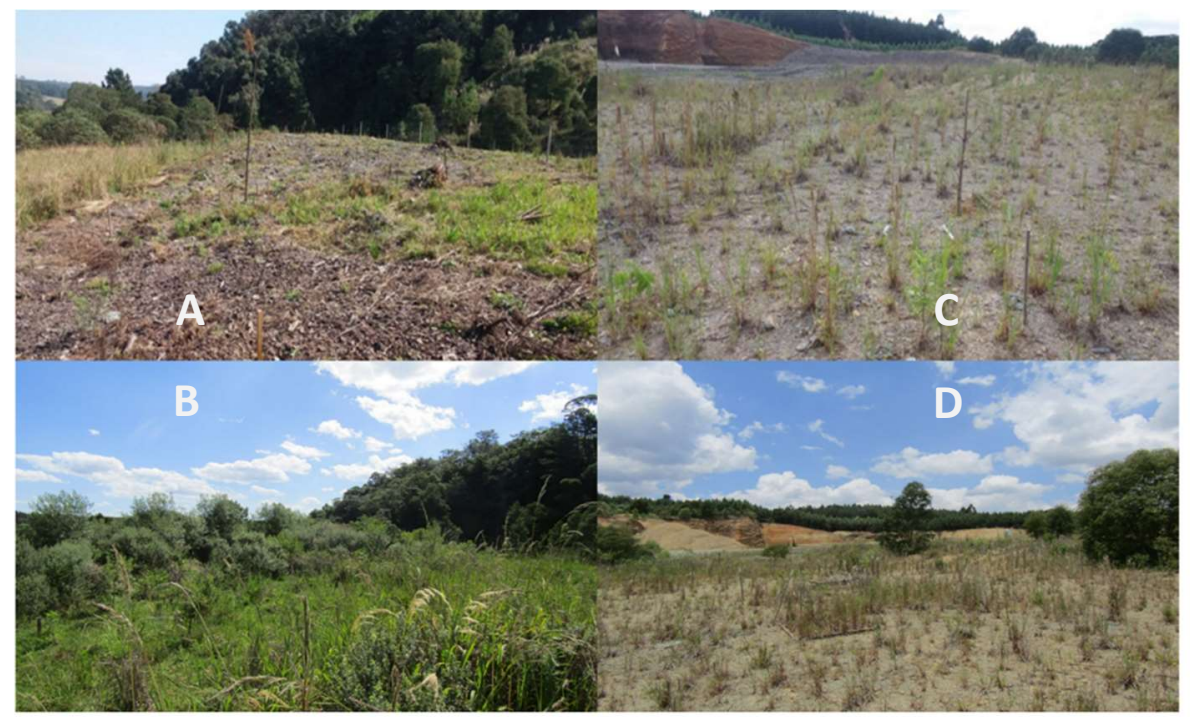

Figura 2: A) Visualização da área Lauro em 2015, ano da deposição do resíduo de origem florestal e da implantação das técnicas de nucleação. B) Visualização da evolução do processo de restauração na área Lauro, no ano de 2018. C) Visualização da área Westarp em 2015, ano da implantação das técnicas de nucleação. D) Visualização da evolução do processo de restauração na área Westarp, no ano de 2018.

\section{CONCLUSÕES}

Mesmo com condições distintas as áreas, após três anos, estão evoluindo no processo de restauração 
e que a utilização do resíduo de origem florestal é um método eficiente e atua de forma positiva no ambiente. Porém, é importante destacar que a aplicação das técnicas de nucleação são as principais responsáveis pela constante evolução dessas áreas, visto que, $84 \%$ e $75 \%$ dos indivíduos encontrados são provenientes destas técnicas, na Westarp e Lauro, respectivamente. Assim, a junção do uso do resíduo de origem florestal com as técnicas nucleadoras são metodologias promissoras para a restauração de áreas mineradas por cascalho no Sul do Brasil, sendo necessários mais estudos para confirmar sua aplicabilidade.

AGRADECIMENTOS: Agradecemos à Klabin S.A. pela disponibilização das áreas estudadas e pelo financiamento deste projeto de pesquisa. Agradecemos também à FAPESC pelo apoio por meio do Programa de Apoio à Pesquisa/Universidade do Estado de Santa Catarina. Por fim, agradecemos ao Grupo de Pesquisa em Gestão de Recursos Florestais da Universidade do Estado de Santa Catarina (UDESC).

\section{REFERÊNCIAS}

ALVARES, C. A.; STAPE, J. L.; SENTELHAS, P. C.; GONÇALVES, J. L. M.; SPAROVEK, G.. Koppen's climate classification map for Brazil. Meteorologische Zeitschrift, v.22, p.711-728, 2013. DOI: http://doi.org/10.1127/0941-2948/2013/0507

BECHARA, F. C.; CAMPOS FILHO, E. M.; BARRETO, K. D.; GABRIEL, V. A.; ANTUNES, A. Z.; REIS, A.. Unidades demonstrativas de Restauração ecológica através de técnicas nucleadoras de Biodiversidade. Revista Brasileira de Biociências, Porto Alegre, v.5, n.1, p.9-11, 2007.

BECHARA, F. C.; REIS, A.; TRENTIN, B. E.. Invasão biológica de Pinus elliottii var. elliottii no Parque Estadual do Rio Vermelho, Florianópolis, SC. Floresta, Curitiba, v.44, p.63-72, 2014. DOI: http://doi.org/10.5380/rf.v44i1.32013

BECHARA, F. C.; DICKENS, S. J.; FARRER, E. C.; LARIOS, L.; SPOTSWOOD, E. N.; MARIOTTE, R.; SUDING, K. N.. Neotropical rainforest restoration: comparing passive, planting and nucleation approaches. Biodiversity and Conservation, Sacramento, v.25, n.11, p.2021-2034, 2016. DOI: http://doi.org/10.1007/s10531-016-1186-7

BRANCALION, P. H. S.; VIANI, R. A. G.; RODRIGUES, R. R.; GANDOLFI, S.. Avaliação e monitoramento de áreas em processo de restauração. In: MARTINS, S. V.. Restauração ecológica de ecossistemas degradados. Viçosa: UFV, 2012 p.262-293.

BROWER, J. E.; ZAR, J. H.. Field \& laboratory methods for general ecology. 2 ed. lowa: Wm. C. Brown Publishers, 1984.

CORBIN, J. D.; HOLL, K. D.. Applied nucleation as a forest restoration strategy. Forest Ecology and Management, v.265, p.37-46, 2012. DOI:

http://doi.org/10.1016/j.foreco.2011.10.013

DUNAISKI, A.; RODERJAN, C. V.. Composição florística e estrutural de comunidades arbóreas em Floresta Ombrófila Mista degradada pela mineração de calcário (Rio Branco do Sul, PR, Brasil). Acta Biológica Paranaense, Curitiba, v.47, n.3-4, p.85-116, 2018. DOI:

http://doi.org/10.5380/abpr.v47i0.63764
DUNAISKI JUNIOR, A.. Regeneração da floresta ombrófila mista após distúrbio por mineração de calcário em Rio Branco do Sul, PR. Tese (Doutorado em Engenharia Florestal) - Universidade Federal do Paraná, Curitiba, 2015.

EMBRAPA. Empresa Brasileira de Pesquisa Agropecuária. Sistema brasileiro de classificação de solos. 3 ed. Brasília: EMBRAPA, 2013.

ENGEL, V. L.; PARROTTA, J. A.. Definindo a restauração ecológica: tendências e perspectivas mundiais. In: KAGEYAMA, P. Y. et al. Restauração ecológica de ecossistemas naturais. Botucatu: Fundação de Estudos e Pesquisas Agrícolas e Florestais, 2003.

ESPÍNDOLA, M. B.; BECHARA, F. C.; BAZZO, M. S.; REIS, A.. Recuperação ambiental e contaminação biológica: aspectos ecológicos e legais. Biotemas, Florianópolis, v.18, n.1, p.2738, 2005. DOI: http://doi.org/10.5007/\%25x

GANN, G. D.; MCDONALD, T.; WALDER, B.; ARONSON, J.; NELSON, C. R.; JONSON, J.; HALLETT, J. G.; EISENBERG, C.; GUARIGUATA, M. R.; LIU, J.; HUA, F.; ECHEVERRÍA, C.; GONZALES, E.; SHAW, N.; DECLEER, K.; DIXON, K. W.. International principles and standards for the practice of ecological restoration. Restoration Ecology, v.27, p.1-4, 2019. DOI: http://doi.org/10.1111/rec.13035

HOBBS, R. J.; NORTON, D. A.. Ecological filters, thresholds, and gradients in the resistance to ecosystem reassembly. In: TEMPERTON, V. M. et al. Assembly rules and restoration ecology. Washington: Island Press, 2004. p.73-95.

IBGE. Instituto Brasileiro de Geografia e Estatística. Manual técnico da vegetação brasileira. 2 ed. Rio de Janeiro: Fundação Instituto Brasileiro de Geografia e Estatística, 2012.

MARCUZZO, S. B.; GANADE, G.; ARAÚJO, M. M.; MUNIZ, M. F. B.. Comparação da eficácia de técnicas de nucleação para restauração de área degradada no Sul do Brasil. Floresta, Curitiba, v.43, n.1, p.39-48, 2013. DOI: 


\section{http://doi.org/10.5380/rf.v43i1.28680}

NERI, A. V.; SOARES, M. P.; NETO, J. A. A. M.; DIAS, L. E.. Espécies de cerrado com potencial para recuperação de áreas degradadas por mineração de ouro, Paracatu-MG. Revista Árvore, Viçosa, v.35, n.4, p.907-918, 2011. DOI: http://doi.org/10.1590/S0100-67622011000500016

PEREIRA, I. M.; GONZAGA, A. P. D.; MACHADO, E. L. M.; OLIVEIRA, M. L. R.; MARQUES, I. C.. Estrutura da vegetação colonizadora em ambiente degradado por extração de cascalho em Diamantina, MG. Pesquisa Florestal Brasileira, v.35, n.82, p.77-88, 2015. DOI:

http://doi.org/10.4336/2015.pfb.35.82.769

R DEVELOPMENT CORE TEAM.. R: A language and environment for statistical computing. R Foundation for Statistical Computing. 2019.

RECH, C. C. C.; SILVA, A. C.; HIGUCHI, P.; SCHIMALSKI, M. B.; PSCHEIDT, F.; SCHMIDT, A. B.; ANSOLIN, R. D.; BENTO, M. A.; MISSIO, F. F.; LOEBENS, R.. Avaliação da Restauração Florestal de uma APP Degradada em Santa Catarina. Floresta e Ambiente, Seropédica, v.22, p.194-203, 2015. DOI: http://doi.org/10.1590/2179-8087.083414

REIS, A.; BECHARA, F. C.; TRES, D. R.; TRENTIN, B. E. Nucleação: concepção biocêntrica para a restauração ecológica. Ciência Florestal, Santa Maria, v.24, p.509-519, 2014. DOI: http://doi.org/10.5902/1980509814591

OKSANEN, J.; BLANCHET, G.; FRIENDLY, M.; KINDT, R.; LEGENDRE, P.; MCGLINN, D.; MINCHIN, P. R.; O'HARA, R. B.; SIMPSON, G. L.; SOLYMOS, P.; STEVENS, M. H. H.; SZOECS, E.; WAGNER, E.. Vegan: community ecology package. 2019.

OLIVEIRA, T. J. F.; BARROSO, D. G.; ANDRADE, A. G.; FREITAS, I. L. J.; AMIM, R. T.. Banco de sementes do solo para uso na recuperação de mata ciliares degradadas na região noroeste fluminense. Ciência Florestal, Santa Maria, v.28, n.1, p.206217, 2018. DOI: http://doi.org/10.5902/1980509831653
SCHICK, J.; BERTOL, I.; COGO, N. P.; GONZÁLEZ, A. P.. Erosividade da precipitação em Lages, Santa Catarina, Brasil. Revista Brasileira de Ciência do Solo, v.38, p.1890-1905, 2014. DOI: http://doi.org/10.1590/S0100$\underline{06832014000600024}$

SILVA, A. C.; HIGUCHI, P.; AGUIAR, M. D.; NEGRINI, M., NETO, J. F.; HESS, A. F.. Relações florísticas e fitossociologia de um Floresta Ombrófila Mista Montana secundária em Lages, Santa Catarina. Ciência Florestal, Santa Maria, v.22, n.1, p.193-206, 2012. DOI: http://doi.org/10.5902/198050985091

STARR, C. R.; CORRÊA, R. S.; FILGUEIRAS, T. S.; HAY, J. D. V.; SANTOS, P. F.. Plant colonization in gravel mine revegetated with Stylosanthes spp. in a Netropical Savanna. Landscape Ecol Eng, v.9, p.189-201, 2012. DOI: http://doi.org/10.1007/s11355-012-0196-1

SER. Society for Ecological Restoration International. Princípios da SER Internacional sobre a restauração ecológica. 2004.

TRENTIN, B. E.; ESTEVAN, D. A.; ROSSETTO, E. F. S.; GORENSTEIN, M. R.; BRIZOLA, G. P.; BECHARA, F. C.. Restauração florestal na Mata Atlântica: passiva, nucleação e plantio de alta diversidade. Ciência Florestal, Santa Maria, v.28, n.1, p.160-174, 2018. DOI: http://doi.org/10.5902/1980509831647

VOGEL, H. F.; CAMPOS, J. B.; BECHARA, F. C.. Early bird assemblages under different subtropical forest restoration strategies in Brazil: passive, nucleation and high diversity plantation. Tropical Conservation Science, v.8, p.912-939, 2015. DOI: http://doi.org/10.1177/194008291500800404

ZANIN, A.; LONGHI-WAGNER, H. M.. Revisão de Andropogon (Poaceae - Andropogoneae) para o Brasil. Rodriguésia, v.62, p.171-202, 2011. DOI: http://doi.org/10.1590/2175$\underline{7860201162112}$

A CBPC - Companhia Brasileira de Produção Científica (CNPJ: 11.221.422/0001-03) detém os direitos materiais desta publicação. Os direitos referem-se à publicação do trabalho em qualquer parte do mundo, incluindo os direitos às renovações, expansões e disseminações da contribuição, bem como outros direitos subsidiários. Todos os trabalhos publicados eletronicamente poderão posteriormente ser publicados em coletâneas impressas sob coordenação da Sustenere Publishing, da Companhia Brasileira de Produção Científica e seus parceiros autorizados. Os (as) autores (as) posteriormente ser publicados em coletâneas impressas sob coordenação da Sustenere Publishing, da Companhia Brasileira de Produção Cientifica e seus parce
preservam os direitos autorais, mas não têm permissão para a publicação da contribuição em outro meio, impresso ou digital, em português ou em tradução. 